\title{
EDITORIAL
}

\section{Phänomenologie als Antwort und Verantwortung. Von Husserl bis Derrida}

Verantwortung war von Anfang an ein zentrales Thema von Husserl und der posthusserlschen Phänomenologie. Viele - auch wenn sie keine Husserl-Spezialisten sind - kennen seine Äußerung über die Philosophen als "Funktionäre der Menschheit", welche die Verantwortung für das "wahre Sein" und die Verwirklichung der Menschheit tragen (Hua VI, 15). Dieser Satz stammt bekanntlich aus der berühmten Schrift Die Krisis der europäischen Wissenschaften und die transzendentale Phänomenologie (1934). Es wäre jedoch falsch anzunehmen, dass der Gedanke der engen Verbundenheit zwischen Philosophie und Verantwortung erst in Husserls Spätwerk in den Vordergrund trat, während das Frühwerk den phänomenologischen Philosophen als "reinen Theoretiker" ausweist. ${ }^{1}$ Wie sehr auch sonst Husserl seine Positionen immer weitergedacht, überdacht und in Frage gestellt hat, so sehr gibt es ein Anliegen, dem er in allen Phasen seines Werks treu geblieben ist, nämlich der dem Begründung eines philosophischen Neuanfangs als Antwort auf die Krise des Wissens. Darin hat er, von Anfang an, die verantwortungsvolle Aufgabe der Phänomenologie und den Beruf des "wahren Philosophen" gesehen. Ich werde hier in groben Zügen die Entstehung und Entwicklung dieser Auffassung skizzieren, um aufzuzeigen wie sich die Verantwortungsproblematik in der post-husserlschen Phänomenologie weiterentfaltet hat. Dabei werde ich auch auf die hier, in dieser Labyrinth-Nummer veröffentlichten Artikel hinweisen, die sich mit diesem mannigfaltigen Gedankengut aus heutiger Sicht beschäftigen.

\footnotetext{
${ }^{1}$ Dies scheint bei Jan Patočka der Fall zu sein. Patočka unterscheidet drei Phasen in der Entwicklung von Husserls Phänomenologie und zeigt durch den Vergleich von Zitaten die Gegensätze zwischen der ersten, der frühen Phase, und den späteren zwei auf. Dabei weist er darauf hin, dass für den frühen Husserl der Philosoph als transzendentaler Beobachter, der auf die absoluten Gegebenheiten reflektiert, "reiner Theoretiker" ist, während der späte Husserl behaupte, dass "der Philosoph, der die Reduktion vollzieht, (...) ein freies, für die Verantwortung übernehmendes Wesen" sei (Patočka 1991, 392). Patočkas Zitatvergleiche sind zwar sehr aufschlussreich in Bezug auf Husserls Versuch einer Überwindung des Cartesianismus, müssen aber als Textauszüge verstanden werden, die in ihrem Gesamtkontext genauer zu untersuchen sind, um z.B. die Verantwortung des "reinen Theoretikers" beim frühen Husserl in den Blick zu bekommen.
} 


\section{Die Verantwortung im Kontext der transzendentalen Phänomenologie}

\subsection{Die Verantwortung der Philosophie als ultimatives Wissen}

Der Begriff der Verantwortung findet in Husserls Philosophie Eingang durch die Thematisierung der Krise des Wissens und der Notwendigkeit einer Rehabilitierung der Philosophie, die schon seinen Lehrer Franz Brentano beschäftigt hatte (Brentano 1929, 83-100). Dies wird zum Leitmotiv der bekannten Studie "Philosophie als strenge Wissenschaft" (1911), die den Grundstein der Phänomenologie legt. Zwar verwendet Husserl in dieser Schrift das Wort "Krise" oder "Krisis" nicht, aber er spricht von Not - von philosophischer Not, von Weltanschauungsnot, von Geistesnot und Lebensnot:

Die philosophische Not als Weltanschauungsnot bezwingt uns. Sie wird nur immer gröBer, je weiter der Umkreis positiver Wissenschaften sich dehnt. Die ungeheure Fülle wissenschaftlich "erklärter" Tatsachen, mit denen sie uns beschenken, kann uns nicht helfen, da sie prinzipiell, mit den ganzen Wissenschaften, eine Dimension von Rätseln mit sich führen, deren Lösung uns zur Lebensfrage wird. Die Naturwissenschaften haben uns die aktuelle Wirklichkeit, die Wirklichkeit, in der wir leben, weben und sind, nicht enträtselt, an keinem einzigen Punkte. (...) Die geistige Not unserer Zeit ist in der Tat unerträglich geworden. (...) Naturalisten und Historizisten kämpfen um die Weltanschauung, und doch sind beide von verschiedenen Seiten am Werk, Ideen in Tatsachen umzudeuten und alle Wirklichkeit, alles Leben in ein unverständliches ideenloses Gemenge von „Tatsachen“ zu verwandeln. Der Aberglaube der Tatsache ist ihnen allen gemein. (Hua XXV, 55-56)

Da diese Not von den Wissenschaften stammt, von ihrem "Aberglauben" an die "Tatsachen", die den Sinneszusammenhang des Weltganzen und des Lebens nur verdunkeln, muss die Not durch eine neue Wissenschaft - die Phänomenologie als "philosophische Wissenschaft" überwunden werden. Diese unterscheidet sich nicht nur von den positiven Wissenschaften, sondern auch von den Weltanschauungen und den sogenannten Weltanschauungsphilosophien, die sich als Streben nach Weisheit und vollkommener Tugend und Tüchtigkeit charakterisieren (ebd., 49). Während die Weltanschauungen an die jeweilige Zeit und Kultur gebunden und deshalb auch veränderlich und vergänglich sind, ist die wahre, die philosophische Wissenschaft auf das Ewige hin gerichtet: "Wissenschaft ist ein Titel für absolute, zeitlose Werte", betont Husserl (ebd., 52). "Ihr Mitarbeiter bedarf nicht der Weisheit, sondern theoretischer Begabung. Was er beiträgt, bereichert einen Schatz ewiger Gültigkeiten, welcher der Menschheit zum Segen gereichen muß" (ebd., 59). Im Gegensatz zum Naturalismus und dem Historizismus als Tatsachenwissen und zur Weltanschauung als handlungsorientierter Weisheitslehre, ist die wissenschaftliche Philosophie Wesenslehre bzw. Wesensforschung (ebd., 35, 47). Obwohl sich die Wesensforschung im Bereich der reinen Theorie bewegt und daher 
keine Daseinsforschung darstellt (ebd., 36), ist sie auf die reale Welt der realen Menschen bezogen, durch den Einfluss, den sie ausübt, denn dieser "Schatz ewiger Gültigkeiten" soll der Menschheit zum Segen verhelfen: "...jeder zeitlose Wert, einmal entdeckt, gehört hinfort zum Wertschatze aller weiteren Menschheit und bestimmt offenbar sogleich den materialen Gehalt der Idee der Bildung, Weisheit, Weltanschauung..." (ebd., 52). Der Kampf um die Überwindung der Krise des Wissens, die Husserl als einen Verfall in Tatsachenwissen beschreibt, ist deshalb von zentraler Bedeutung, weil sich hier das Schicksal der Menschheit, bzw. "die Möglichkeit einer stetig fortschreitenden Realisierung der Ewigkeitsidee der Menschheit", entscheidet. "Denn bis zu den weitesten Weiten des ethischen Ideals, bis zu denen, die die Idee der Menschheitsentwicklung bezeichnet, reichen unsere historischen Einflüsse und somit auch unsere ethischen Verantwortungen", betont er (ebd., 54). Mit anderen Worten, die Philosophie als strenge Wissenschaft hat eine höhere Berufung als jede andere Wissenschaft und jede Weltanschauung, da sie allein dazu berufen ist, die "Ewigkeitsidee der Menschheit" aufzuzeigen und daher auch die Verantwortung für deren Verwirklichung trägt.

Dieser Gedanke der philosophischen Verantwortung für die Menschheitsentwicklung, der in "Philosophie als strenge Wissenschaft" (1911) nur ganz allgemein formuliert ist, wird später in Husserls weniger bekannten Abhandlung "Meditation über die Idee eines individuellen und Gemeinschaftslebens in absoluter Selbstverantwortung" (1924) detailliert ausgeführt. Husserl erläutert hier zu Beginn den Bezug zwischen Theorie und Praxis und stellt die These auf, dass je grundlegender und universaler die Erkenntnis ist, desto höher auch die Verantwortung. Er betont, dass sich in den Erkenntnisformen der theoretischen Wahrheit alle anderen Wahrheiten, so jede Wertwahrheit und praktische Wahrheit, in prädikativen Formen ausspricht, bestimmt und auch erkenntnismäßige Begründungsformen annimmt. Zwar bekunde sich die Wahrheit, die Echtheit des Wertes und des Werkes ursprünglich naiv im Gemüt, "aber verantwortet wird letztlich die Echtheit der Wahrheit des Wertes im Erkennen, das in Urteilseinstellung und in seinen logischen Formen über Wert und Unwert prädiziert und einsichtig die zufällig vorliegende Wertanschauung auf generell einsichtige Wertnormen zurückbezieht und dadurch eine höhere Verantwortung, als Erkenntnisverantwortung, gewinnt. Die höchste und letzte Verantwortung aber entspringt in der Erkenntnis aus transzendentaler Einstellung auf die letztkonstitutiven Gemüts- und Willensleistungen." (Hua VIII, 194)

Diese "höchste Verantwortung" der transzendentalen Phänomenologie komme ihr zu, so Husserl, weil sie als grundlegende universale Wissenschaft, als eine Theorie aller Theorien, alle Erkenntnis überhaupt in sich birgt und systematisch entwickelt.

Damit gewinnt aber auch der Gedanke der Verantwortung für diese universale Wissenschaft eine erhöhte Bedeutung. Denn gewinnen alle anderen Theorien, welcher Einzeldisziplinen immer, in ihr letzte und vollendete Rechtfertigung, so ist sie selbst nicht in 
der glücklichen Lage, wie diese über sich noch eine Instanz zu haben, an die sie sich ihrerseits zu diesem Behufe wenden könnte, sondern als die Theorie aller Theorien ist sie auf sich selbst zurückgewiesen und zurückbezogen; alles, was sie aufstellt, muß aus ihr selbst heraus nachher seine Rechtfertigung finden, und so befindet sie sich allerdings in einem Zirkel, ( ...) dem gemäß sie nicht nur Verantwortung trägt für alle anderen Disziplinen, sondern vor allem Selbstverantwortung im radikalsten und absoluten Sinn. (Hua VIII, 195-196)

Dieser Kreislauf der transzendentalen Phänomenologie zwischen Verantwortung für alle anderen Wissenschaften und Disziplinen einerseits und absoluter und radikaler Selbstverantwortung andererseits wird von Husserl noch zusätzlich durch die gesellschaftliche Rolle und Mission, welche die Philosophie als ultimatives Wissen in der Geschichte der Menschheit zu spielen hat, erweitert. Dies wird durch seine Konzeption des Telos des Wissens und des Telos des individuellen und gemeinschaftlichen Lebens, die eng miteinander verbunden sind und einen analogen Kreislauf bilden, veranschaulicht.

Der teleologische Charakter der universalen Philosophie wird durch die These begründet, dass sie als Allwissenheit ein im Unendlichen liegendes Ziel ist, das nicht von einem Philosophen allein, noch von irgendeiner philosophischen Gemeinschaft erreichbar ist. Diese Allwissenheit als "universale Einheit alles Wissens" ist das Telos, "die absolute Endidee, die jeden Philosophen und jede Philosophengemeinschaft leitet" (ebd., 196). Diese sich ins Unendliche erstreckende stufenweise Vervollkommnung des Wissens dürfe zwar nicht als historisches Faktum, sondern nur als Ideal gedacht werden und dennoch sei sie nicht bloß eine theoretische Liebhaberei der Menschheit, denn sie ermögliche dem Einzelnen ein Leben in absoluter Selbstverantwortung:

Wenn wir bedenken, daß jede Art menschlichen Tuns, Wollens und Fühlens Gegenstand von Wissenschaften werden kann, in denen es zum theoretischen Thema gemacht wird, und wenn wir ferner bedenken, daß jede theoretische Erkenntnis alsbald eine normative Wendung erfahren kann, nach der sie zur Regel für eine mögliche Praxis usw. wird, dann sehen wir ein, daß Philosophie - als universale Wissenschaft, berufen, den Urquell abzugeben, aus dem alle Wissenschaften ihre letzte Rechtfertigung schöpfen - wir sehen ein, daß eine solche Philosophie keine theoretische Liebhaberei der Menschheit sein kann, daß ein philosophisches Leben vielmehr verstanden werden muß als ein Leben überhaupt aus absoluter Selbstverantwortung: das personale Einzelsubjekt, als Subjekt personalen Lebens, will in all seinem Leben, in all seiner Praxis sich wahrhaft frei entscheiden, d.i. so, daß es jederzeit das Recht seiner Entscheidung vor sich selbst verantworten kann. (ebd., 197)

Da aber das Einzelsubjekt immer ein Glied einer Gemeinschaft ist, so haben wir es laut Husserl mit einem komplexen Geflecht von individueller und kollektiver Selbstverant- 
wortung zu tun. Denn einerseits könne sich die Gemeinschaft nur im einzelnen Subjekt verantworten, andererseits aber sei die Selbstverantwortung des Einzelnen, der sich "als Glied und Funktionär der Gemeinschaft weiß", auch eine Verantwortung für die Gemeinschaft selbst. Dadurch, dass die Gemeinschaft eine Synthesis der einzelnen Individuen durch interpersonale Intentionalität, d.h. "eine durch das soziale Füreinander- und Ineinander-Leben und -Wirken gestiftete Einheit" sei, bilde auch die Selbstverantwortung für eine Gemeinschaft eine Synthesis, die die einzelnen Selbstverantwortungen intentional ineinander verflicht und zwischen ihnen eine innere Einheit herstelle. Somit wird die Selbstverantwortung zu einer Mit-Verantwortung für die Gemeinschaft, aber auch zu einer Verantwortung für die Selbstverantwortung der Anderen, die ich für ein selbstverantwortliches Leben gewinnen muss und zur Verantwortung ziehen kann, wenn sie dagegen verstoßen:

Und wenn ich schon in mir die Notwendigkeit erkannt habe, Selbstverantwortung zu vollziehen und für mich ein Leben aus universaler und absoluter Selbstverantwortung zu wählen und dafür die normative Idee zu entwerfen, so kann ich und werde ich erkennen, daß, was für mich, für jedermann das Beste wäre und die Forderung eines solchen philosophischen Lebens von mir an jeden und von jedem an sich selbst zu stellen ist; auch daß ich jeden dazu zu bestimmen versuchen muß und ihn dafür verantwortlich machen, daß er sich für ein solches Leben entscheide und entsprechend lebe." (ebd., 199)

In diesem Kontext ergeben sich für Husserl mehrere Fragen, nämlich: Wie würde diese ideelle Gemeinschaft aussehen? Wie kann man eine solche Gemeinschaft gerichteter Willen auf ein Leben aus absoluter Verantwortlichkeit aufbauen? Ist überhaupt ein solches Leben möglich, ohne dass zuvor erkenntnismäßig seine Idee und damit eine normative Wissenschaft davon, d.h. eine Ethik, entworfen wäre? Die Antworten die Husserl gibt, zeigen noch klarer den Bezug zwischen Theorie und Praxis. Das, was dabei herauskommt und herauskommen soll, ist die fundamentale Rolle der Erkenntnistheorie als philosophischer Disziplin, die den Grund für normative und axiologische Urteile bildet, die wiederum für den Willen, das Handeln und das Leben aus bewusster Eigenverantwortung ausschlaggebend sind. Deshalb ist es kein Zufall, dass Husserl die ideale Form der Gemeinschaft in axiologischen Begriffen beschreibt, die den Wert der Gemeinschaft vom Wert der wissenschaftlichen, bzw. der philosophischen Erkenntnis abhängig macht:

Die höchste axiologische Gestalt einer Gemeinschaft, oder die in absoluter Vernunfterkenntnis als höchstwertig zu erkennende Gemeinschaft, wäre eine solche Gemeinschaft, die diese Vernunfterkenntnis selbst in sich trüge, die diese absolute Wertung in sich selbst vollzöge, also ihres absoluten Wertes bewußt wäre, und die absolut wertvoll aus dem Willen heraus wäre, eine absolut werte zu sein. (ebd., 200) 
Für Husserl besteht die Aufgabe der Philosophie darin, die Idee dieser Gemeinschaft bewusst zu machen, indem ihre notwendigen Entwicklungsstadien aufgezeigt werden. Diese Stadien wären, erstens, die vorwissenschaftlichen Motive, die die Idee in den Blick treten lassen, wie z.B. die vorbildlichen Handlungen und Persönlichkeiten, die zunächst Nachahmung und später wahre Nachfolge finden; zweitens, die Begründung von Normen und normative Regeln; drittens, das Beschließen von Gesetzen. Damit wird die Philosophie als universale Erkenntnis und einheitliche Wissenschaft in den Dienst der Gemeinschaft gestellt:

Erkenntnisvernunft ist Funktion der praktischen Vernunft, der Intellekt ist Diener des Willens. Aber der Diener vollzieht in sich selbst Willensfunktionen, gerichtet auf Erkenntnisgebilde selbst, die eben notwendige Mittel sind, den Willen überall zu leiten, ihm rechte Ziele und Wege zu zeigen. Das Erkennen wollen ist vorausgesetzt für alles andere Wollen, wenn dieses die höchste Wertform besitzen soll. (ebd., 201)

Doch Husserl ist sich zugleich bewusst, dass der Weg zur Vervollkommnung der Menschheit nie zu einer wirklich vollkommenen Menschheit führen wird. Daher wird auch die Gefahr einer Krise, eines wissenschaftlichen und moralischen Verfalls immer gegeben sein:

Die Wissenschaft rechnet nicht bloß aus, und etwa gar ein für allemal, was das Willenssubjekt in jedem Falle tun muß, als ob der Wille bloß sein realisierendes Ja zu sagen hätte zu dem eindeutig fest errechneten Erkenntnisresultat. Wesensmäßig bleiben immerzu Spielräume der Unerkanntheit, Unbestimmtheit, der Gefahr, des Irrtums, der Sünde usw. Der unendliche Progressus der Erkenntnis ist ein Progressus der Verengung der Schranken und der Gefahren, aber er ist unendlicher Progressus, und ins Unendliche bleibt die Gefahr, Sünde usw." (ebd., 202-203)

Diese Unvollkommenheit des menschlichen Seins, die sich im Gemeinschaftsleben widerspiegelt und die Husserl indirekt anspricht, erinnert an das später entwickelte Konzept der Ambiguität der "menschlichen Realität" bei Jean-Paul Sartre und Simone de Beauvoir (Sartre, Beauvoir), sowie an die "ontologische Disproportion" als Fehlbarkeit des Menschen bei Paul Ricœur (Ricœur 1971, 18). Die Unvollkommenheit, von der Husserl spricht und die sein Verantwortungskonzept stark prägt, hat jedoch keinen ontologischen, bzw. anthropologischen, oder moralischen Grund, sondern einen gnoseologischen. Wie Philippe Buckley in seinem in dieser Labyrinth-Nummer veröffentlichten Artikel zeigt, unterscheidet Husserl zwei Typen von Erkenntnis - die eigentliche oder unmittelbare intuitive Wesenserkenntnis und die uneigentliche, die durch Zeichen und symbolische Vorstellungen vermittelt wird (Buckley 2016, 32ff). Zur Letzteren gehören auch die berechnende Vernunft und die Tatsachenwissenschaften. Wenn also Husserl die Abhandlung damit beendet, dass die (eigentliche) Wissenschaft nicht bloß berechnet, um dem wollenden und handelnden Subjekt dann die "richtige" Handlungsweise vorzuschreiben, dann will er damit mehrere Dinge klarstellen. Erstens, dass 
es zum Wesen der Erkenntnis dazu gehört gewisse Irrwege zu gehen, zweitens, dass man deswegen der universalen Philosophie als transzendentale Phänomenologie bedarf, um die Irrtümer und das Unwissen möglichst zu begrenzen, und, drittens, dass es "Spielräume" der Unbestimmtheit und Unerkanntheit gibt, welche die Gefahr eines Rückfalls oder Verfalls in Krisen des Wissens und des praktischen Lebens nie beseitigen werden können. Diese Unbestimmtheit oder Ungewissheit, die bei Husserl nur indirekt mit dem Problem der Verantwortung in Zusammenhang gebracht wird, bekommt später bei Heidegger und Derrida einen wichtigen Platz (siehe Derrida 1986, 171).

\subsection{Verantwortung im Zeichen der Europäischen Geistesgeschichte}

Während die Abhandlung "Meditation über die Idee eines individuellen und Gemeinschaftslebens in absoluter Selbstverantwortung" (1924) die idealen Konturen der universalen Philosophie als teleologischer Ewigkeitsidee der Menschheit entwirft, ist der zehn Jahre später in Wien gehaltene Vortrag "Die Krisis des Europäischen Menschentums und die Philosophie" (1935) $)^{2}$ und das darauf folgende Werk Die Krisis der europäischen Wissenschaften und die transzendentale Phänomenologie (1935/36) auf die historischen Entwicklungsformen dieser teleologischen Idee ausgerichtet, wobei das spezifisch "Europäische" besonders herausgearbeitet und artikuliert wird. Die Frage, die sich hier stellt ist - wie Paul Ricœur es treffend formuliert hat - Wie kam es das ausgerechnet "der ahistorischste Professor" sich den Fragen der Geschichte und der Philosophie der Geschichte zuwandte? (Ricœur 1986, 22) Ricœur beantwortet diese Frage indem er auf die geschichtliche Situation, in der sich Husserl in den 1930er Jahre befand, verweist: Als Philosoph nichtarischer Herkunft, der den Nazis suspekt war und durch Pensionierung zum Schweigen gebracht wurde, musste Husserl feststellen, dass der Geist selbst eine Geschichte hat, dass sich diese für ihn als eine Gefahr, als eine Krankheit erweisen kann, die ihn sogar umzubringen vermag.

Durch das Bewusstsein der Krise wurde man in der Epoche des Nationalsozialismus tatsächlich in die Geschichte hineingeholt: die Ehre des Rationalismus verlangte, dass man sagt, wer der Kranke war, d.h. worin der Sinn und der Unsinn des Menschen bestand. (Ricœur, ebd.) ${ }^{3}$

Ein anderer Grund für Husserls Zuwendung zu diesen geschichtlichen Problemen, den ich hier zusätzlich anführen möchte, besteht darin, dass er den Vortrag auf Einladung des

\footnotetext{
${ }^{2}$ Der ursprüngliche Titel des Vortrags war "Die Philosophie in der Krisis de europäischen Menschheit". Husserl hielt den Vortrag auf Einladung des Wiener Kulturbundes am 7. Und 10. Mai 1935.

${ }^{3}$ Die Übersetzung ist von mir, Y.R. Im Original heißt es: "c'était par la conscience de crise qu'à l'époque $\mathrm{du}$ national-socialisme on entrait en fait dans l'histoire : pour l'honneur du rationalisme il s'agissait de dire qui était malade, donc ou était le sens de l'homme et ou le non-sens" (Ricœur 1986, 22).
} 
Österreichischen Kulturbundes ${ }^{4}$ vorbereitet hatte, für den die Krisis- und Europathematik im Zentrum stand. Für den Kulturbund ging es darum, "in einer Zeit der Verwirrung, des Zusammenbruchs und des Verlustes vertrauter Traditionen (...) sich der 'alten' Werte zu besinnen und alle geistige und kulturelle Potenz zur Überwindung der Krise einzusetzen" (Malina 1986, 261). Dies sollte auf zweifache Weise geschehen, zum einen durch die "Zusammenfassung der für Europa innerlich verantwortlichen Europäer" und zum anderen durch die Schaffung einer Gemeinschaft, die "über Grenzen von Nation und Weltanschauung hinweg die europäischen Geister verbindet" (ebd., 265). Besinnung auf "geistige Werte", auf die führende Rolle der Kulturträger (der Intellektuellen) und ihre spezielle Verantwortung, auf die Geschichte und die Zukunft Europas, waren die zentralen Themen, die im damaligen "Zeitgeist" lagen und zwar nicht nur in Deutschland, sondern auch in Frankreich ${ }^{5}$. So war es auch kein Zufall, dass die Zeitschrift des Österreichischen Kulturbundes den Namen Zeitgeist gewählt hatte. In diesem thematischen und geschichtlichen Kontext war Husserls Vortrag "Die Philosophie in der Krisis der europäischen Menschheit" nicht nur willkommen, sondern stieß auf ein so reges Interesse, das er selbst nicht erwartet hatte. Husserl beschreibt das Ziel seines Vortrags in folgender Weise:

Ich will in diesem Vortrage den Versuch wagen, dem so viel verhandelten Thema der europäischen Krisis ein neues Interesse dadurch abzugewinnen, daß ich die geschichtsphilosophische Idee (oder den teleologischen Sinn) des europäischen Menschentums entwickle. Indem ich dabei die wesentliche Funktion aufweise, welche in diesem Sinn die Philosophie und ihre Verzweigungen als unsere Wissenschaften zu üben haben, wird auch die europäische Krisis eine neue Erleuchtung gewinnen. (Hua VI, 314)

Husserl ist sich im Klaren, dass das Thema der europäischen Krisis auch im Kulturbund schon viel debattiert wurde und so setzt er den Akzent auf die teleologische Sinnentwicklung und Zielgerichtetheit der europäischen Geistesgeschichte, die er mit der Philosophiegeschichte identifiziert. Die unmittelbare Konsequenz dieser Identifikation wird schon zu Beginn des Vortrages unmissverständlich formuliert: Wollen wir die geistige Krise unserer Zeit verstehen

\footnotetext{
${ }^{4}$ Der Österreichische Kulturbund wurde 1922 gegründet als "Vereinigung der geistigen Führer und Träger der Kultur, um unabhängig von Partei, Klasse und Rasse und mit Ausschluss jeder Politik schöpferische Beziehungen zwischen den verschiedenen Standpunkten und Auffassungen zu ermöglichen" (Malina 1986, 259). Die Zielsetzungen des Vereins wurden später jedoch der Kulturpolitik des Austrofaschismus angepasst. Nach der deutschen Okkupation Österreichs in 1938 und dem Gesetz über die Überleitung und Eingliederung von Vereinen und Organisationen (GBI. Nr. 136/1938) wurde der Kulturbund im selben Jahr aufgelöst (ebd., 255).

${ }^{5}$ Zur Erinnerung: der französische Personalismus und seine Zeitschrift Esprit (Geist), die 1932 von Emmanuel Mounier gegründet worden war, verstanden sich als eine Antwort auf die "ökonomische und geistige Krise" bzw. als geistige Erneuerung durch die s.g. "personalistische und gemeinschaftliche Revolution" (Mounier 1962/III, 183, idem 1961/I, 129-220).
} 
bzw. "das kranke Europa" heilen, dann müssen wir uns der Geschichte der Philosophie zuwenden, aus der heraus allein der Sinn unseres eigenen, individuellen und gemeinschaftlichen Lebens zu verstehen ist. Husserl postuliert jedoch nicht einfach diese Identifikation, sondern versucht sie durch den Zusammenhang zwischen Leben, Welt und Geist, bzw. durch die Einführung seines bekannt gewordenen Konzepts der Lebenswelt, sichtbar zu machen. Denn unser Leben, das sich in der Welt vollzieht, in eine Welt, die eben in Krise steckt, ist nicht schlicht ein natürliches Phänomen im Sinne einer objektiven Natur, die körperlich erkranken kann, sondern einer Natur so wie die Griechen sie verstanden, nämlich, einer sich in der Geistigkeit abspielender Tatsache. So ist auch unsere Umwelt ein geistiges Gebilde, "ein Begriff, der ausschließlich in der geistigen Sphäre seine Stelle hat" (ebd., 317). Durch diese These deutet Husserl zugleich an, worin die Wurzel der Krise eigentlich besteht: "Natur als in sich Geistesfremdes anzusehen und demzufolge Geisteswissenschaft durch Naturwissenschaft unterbauen und so vermeintlich exakt machen zu wollen, ist ein Widersinn" (ebd.). Anders gesagt, man habe vergessen, was die Griechen schon wussten, nämlich, dass die Erkenntnis der Natur eine geisteswissenschaftliche Leistung ist. Dies zu untersuchen hieße die tiefsten Gründe des Ursprungs des verhängnisvollen Naturalismus aufzuzeigen, damit der eigentliche Sinn der Krisis des europäischen Menschentums an den Tag kommen könne. Husserl geht deswegen von der Frage aus: Was charakterisiert die geistige Gestalt Europas? Es handle sich seiner Meinung nach um eine neue Menschheitsepoche, "die nunmehr bloß leben will und leben kann in der freien Gestaltung ihres Daseins, ihres historischen Lebens, aus Ideen der Vernunft, aus unendlichen Aufgaben" (Hua VI, 319). Diese neue Epoche sei die der Geburt der (europäischen) Philosophie als theoretischer Einstellung, die bei den Griechen ihren historischen Anfang habe (ebd., 326). Mit den ersten theoretischen Konzeptionen der alten Griechen sei der Mensch allmählich zu einem neuen Menschen geworden. Sein geistiges Sein sei dadurch in die Bewegung einer fortschreitenden Neubildung getreten, das zu einem neuen Stil personalen Daseins in seinem Lebenskreis geführt habe. Damit sei dann auch eine neue Weise der Vergemeinschaftung als Ideenerzeugung und idealer Normierung vergemeinschafteten Lebens entstanden:

Das also vollzieht sich zunächst im geistigen Raum einer einzigen, der griechischen Nation, als Entwicklung der Philosophie und der philosophischen Gemeinschaften. In eins damit erwächst zunächst in dieser Nation ein allgemeiner Kulturgeist, das ganze Menschentum in seinen Bann ziehend, und ist so eine fortschreitende Verwandlung in Form einer neuen Historizität (ebd., 322-323).

Durch die Philosophie sei so ein neuer Geist der Normierung in Bezug auf unendliche Aufgaben und Ideale für die Menschheit geschaffen worden, der die Nationen zu einem übernationalen Ganzen, zu einer Art "Allsozietät" erheben solle. Darin bestehe die "archontische" 
Funktion der Philosophie, ihre besondere Aufgabe und Verantwortung für die Wahrung und Weiterentwicklung der Ideale und des Allideals als Universum aller Normen (ebd., 336).

In der späteren Version - die Krisis der europäischen Wissenschaften und die transzendentale Phänomenologie - zieht Husserl einen großen historischen Bogen von der griechischen Philosophie durch die Neuzeitliche, um zu zeigen wie es dazu kam, dass das in der Antike aufgebrochene Telos sich verirrte und es zu einer geistigen Krise gekommen ist, trotz der großen wissenschaftlichen Fortschritte (Hua VI, 12-123). In dem Wiener Vortrag ist dieser Verfall zwar kurz, jedoch sehr klar formuliert. Die Krisis ist, so Husserl, eine "Not der Methode", ein Verfall der Rationalität in Einseitigkeiten wie z.B. die Naivität des Objektivismus mit seiner Naturalisierung des Geistes und Vergessen des Subjekts (ebd., 339).

Durch ihren Objektivismus kann die Psychologie gar nicht die Seele, das ist doch das Ich, das tut und leidet, in seinem eigenwesentlichen Sinn ins Thema bekommen. Sie mag wertendes Erlebnis, Willenserlebnis auf körperliches Leben verteilt objektivieren und induktiv behandeln, kann sie das auch für Zwecke, Werte, Normen, kann sie Vernunft zum Thema machen, etwa als "Disposition"? (...) Immer fühlbarer wird allgemein die Reformbedürftigkeit der ganzen neuzeitlichen Psychologie, aber noch versteht man nicht, daß sie durch ihren Objektivismus versagt hat, daß sie überhaupt an das eigene Wesen des Geistes nicht herankommt, daß ihre Isolierung der objektiv gedachten Seele und ihre psychophysische Umdeutung des In-Gemeinschaft-seins eine Verkehrtheit ist. Gewiß hat sie nicht vergeblich gearbeitet und viele auch praktisch wertvolle empirische Regeln aufgewiesen. Aber sie ist so wenig eine wirkliche Psychologie als die Moralstatistik mit ihren nicht minder wertvollen Erkenntnissen eine Moralwissenschaft ist. (ebd., 343-344)

Daraus folgt für Husserl, dass der Geist sich seiner Aufgaben und Verantwortung bewusst werden kann nur, wenn er aus der naiven Außenwendung zu sich selbst zurückkehrt und bei sich selbst in reiner Selbstbesinnung bleibt. Oder wie er pathetisch abschließt:

Die Krise des europäischen Daseins hat nur zwei Auswege: Den Untergang Europas in der Entfremdung gegen seinen eigenen rationalen Lebenssinn, den Verfall in Geistfeindschaft und Barbarei, oder die Wiedergeburt Europas aus dem Geiste der Philosophie durch einen den Naturalismus endgültig überwindenden Heroismus der Vernunft. (ebd., 347-348)

Diese Konzeption mag gerade heute, wo die Stellung der Philosophie und der Philosophen immer prekärer wird, megaloman erscheinen, sie schreibt sich jedoch in eine lange philosophische Tradition ein. An dieser Stelle sei kurz an zwei paradigmatische Konzeptionen erinnert, die Husserl als Anregung gedient haben könnten, nämlich diejenigen von Plato und Kant. 
Die Idee der Teleologie als Wirkungskraft der Vernunft in Natur und Gesellschaft, die Husserl aufgreift, um die besondere Rolle und die Verantwortung der Philosophen zu argumentieren, lässt sich schon bei Plato, den er immer wieder erwähnt, finden. Indem Plato Anaxagoras' Begriff vom voũ $\varsigma$ aufgreift, entwickelt er die These, dass die Vernunft nicht nur die Welt ordnet, sondern dem Menschen auch das Wissen um das Beste ermöglicht, das wiederum die Verwirklichung des Guten und des Gerechte im Staat fordert (Phaidon 97c-e, 99a). Da die Besten, die das Wissen haben und sich von der Vernunft leiten lassen, die Philosophen sind, sollten alle Herrscher echte Philosophen sein, denn solange die politische Macht und die Philosophie nicht Hand in Hand gehen, solange würde es auch kein Ende der Übel für die Menschheit geben (Politeia 473c-d).

Obwohl Kant, seinerseits, die Idee einer objektiven Teleologie als Zweckausrichtung der Natur ablehnt, wird das Erfassen der Welt als zweckmäßiges Ganzes von ihm als heuristisches Prinzip gebilligt (Kant AA V, 411). Der teleologische Gebrauch der reflektierenden Urteilskraft wird auch auf die Gesellschaft und die menschliche Geschichte angewendet:

Ein philosophischer Versuch, die allgemeine Weltgeschichte nach einem Plane der Natur, der auf die vollkommene bürgerliche Vereinigung in der Menschengattung abziele, zu bearbeiten, muß als möglich und selbst für diese Naturabsicht beförderlich angesehen werden (...) wenn man indessen annehmen darf: daß die Natur selbst im Spiele der menschlichen Freiheit nicht ohne Plan und Endabsicht verfahre. (Kant AA VIII, 029)

Diese teleologische Vorgangsweise, die ihren Anfang in der griechischen Geschichte nimmt, soll als einen Leitfaden dazu dienen, um "ein sonst planloses Aggregat menschlicher Handlungen wenigstens im Großen als ein System darzustellen" (ebd.). Mit anderen Worten, wir finden schon bei Kant den Gedanken, dass die europäische Geschichte und ihr philosophischer Geist mit den Griechen beginnt. Was Husserl betrifft, so äußert er diese Idee bereits in den Vorlesungen Erste Philosophie (1923/24) [siehe Hua VII, 317 und Hua VIII, 3-4], doch sie wird erst im Krisis-Vortrag expliziert und zwar auf eine Art und Weise, die ihm die harten Vorwürfe des "Eurozentrismus" und des "intellektueller Rassismus" eingebracht hat ${ }^{6}$. In diesem Zusammenhang werden immer wieder gewisse Textpassagen zitiert und kommentiert, in denen er Unterschiede zwischen der geistigen Entwicklung bestimmter Ethnien und Nationen annimmt und behauptet, die Europäer hätten die höchste Entwicklungsstufe erreicht, weil sie allein zum theoretischen bzw. philosophischen Denken fähig seien:

Im geistigen Sinn gehören [zur geistigen Gestalt Europas] offenbar die englischen Dominions, die Vereinigten Staaten usw. zu Europa, nicht aber die Eskimos oder Indianer

\footnotetext{
${ }^{6}$ Siehe z.B. Michael Murray. "Husserl and Heidegger: Constructing and Deconstructing Greek Philosophy," Review of Metaphysics, XLI (1988): 501-518, hier 504; Jacques Derrida. De l'esprit. Heidegger et la question. Paris: Éditions Galilée, 1987, 95-96.
} 
der Jahrmarktsmenagerien oder die Zigeuner, die dauernd in Europa herumvagabundieren. (Hua VI, 318-319)

Es liegt darin etwas Einzigartiges, das auch allen anderen Menschheitsgruppen an uns empfindlich ist als etwas, das, abgesehen von allen Erwägungen der Nützlichkeit, ein Motiv für sie wird, sich im ungebrochenen Willen zu geistiger Selbsterhaltung doch immer zu europäisieren, während wir, wenn wir uns recht verstehen, uns zum Beispiel nie indianisieren werden. Ich meine, wir fühlen es (...), daß unserem europäischen Menschentum eine Entelechie eingeboren ist, die den europäischen Gestaltenwandel durchherrscht und ihm den Sinn einer Entwicklung auf eine ideale Lebens- und Seinsgestalt als einen ewigen Pol verleiht. (Hua VI 320)

Man mag hier und dort ein weltumgreifendes Interesse konstatieren, das beiderseits, also auch bei der indischen, chinesischen und ähnlichen "Philosophien" zu universalen Welterkenntnissen führt (...) Aber nur bei den Griechen haben wir ein universales ("kosmologisches") Lebensinteresse in der wesentlich neuartigen Gestalt einer rein "theoretischen" Einstellung, und als Gemeinschaftsform, in der es sich aus inneren Gründen auswirkt, die entsprechende wesentlich neuartige der Philosophen, der Wissenschaftler (der Mathematiker, der Astronomen usw.). Es sind die Männer, die nicht vereinzelt, sondern miteinander und füreinander, also in interpersonal verbundener Gemeinschaftsarbeit, Theoria und nichts als Theoria erstreben und erwirken (Hua VI, 325-326)

Wenn ich diese problematischen Passagen anführe, so nicht deshalb um erneut Vorwürfe anzubringen, sondern um einige Fragen in Bezug auf die Verantwortungsproblematik bei Husserl zu stellen. Was bedeutet es von den Konsequenzen her, wenn wir manchen Ethnien, Gruppen, Nationen usw. eine höhere geistige Entwicklung zusprechen als anderen, denen wir diese absprechen und sie als unmündig erklären? Wie die Nazis in diesem Zusammenhang ihre Verantwortung verstanden ist wohl bekannt: Verantwortung für die Gesundheit und Reinheit der Nation bedeutete für sie nichts anderes als die Aufgabe, alle Nichtarier (Juden, Zigeuner, Slaven usw.), sowie Geistesschwachen zu vernichten. Dieses Verantwortungsverständnis und seine Anwendungsweise kann man keineswegs Husserl anlasten. Da er jedoch die Selbstverantwortung des Philosophen unaufhörlich betonte und noch dazu den Anspruch hatte, die Anderen ein verantwortungsvolles Denken und Handeln zu lehren, fragt man sich natürlich, wie es denn mit seiner eignen Verantwortung stünde in Anbetracht der Vernichtung der Zigeuner durch die Nazis, die kurz nach seinem Tode begann. Was hätte Husserl, falls er noch gelebt hätte, dazu gesagt? Hätte er darauf Bezug genommen oder zumindest etwas in seinen Schriften revidiert? Da man darüber nur spekulieren kann, gilt es die prinzipielle Frage ins Auge zu fassen, ob Husserl den "geistig Überlegenen" (Europäer) nur eine höhere Verantwortung zugesprochen hat oder, ob er meinte, sie sollen auch Verantwortung für die Unmündigen bzw. die "Rückständigen" übernehmen? 
Es besteht kein Zweifel, dass für Husserl die Aufgabe der "wahren Philosophie" mit einer "höchsten" Verantwortung und Selbstverantwortung verknüpft ist: sie ist "eine Frage des wissenschaftlichen Gewissens, das uns aufruft in universaler und radikaler Besinnung, die - in höchster Selbstverantwortung vollzogen - selbst zu wirklicher und höchster Wahrheit werden muß" (Hua VI, 200). Das muss noch nicht bedeuten, dass die Philosophen die Verantwortung für andere übernehmen sollten. Es heißt nur, dass sie sich zuerst der Zweckidee der Philosophie und ihrer Bedeutung für die Menschheit bewusst werden müssen, um dieses Bewusstsein dann weiter zu geben und an die philosophische Verwirklichung dieser Zweckidee als Trägerin des wahren Seins des Menschen und der Gesellschaft weiter zu arbeiten. So weit, so gut. Die Probleme in Husserls Konzept der "geistigen Gestalt" Europas tauchen aber sofort auf, wenn man Husserls These über die gleichen menschlichen Fähigkeiten zu rationalem Denken seiner These gegenüberstellt, dass nur die europäische Menschheit "als Telos bewusst geworden ist" und damit "eine neue, höhere Entwicklungsstufe" eingeleitet hat (Hua VI, 321). Mit anderen Worten, Husserls Verantwortungskonzept kann man zwar nicht vorwerfen, dass die Europäer als geistig Überlegene für "Andere", die auf einer unteren geistigen Stufe stehen, Entscheidungen und Verantwortung übernehmen würden, aber das Konzept bleibt dennoch sehr problematisch, weil diesen "Anderen" die höhere Fähigkeit eines Lebens aus eigener Selbstverantwortung gar nicht zugetraut wird, da sie von vornherein als weniger zivilisiert abgestempelt werden. Diese "Anderen", die Nichteuropäer, können höchstens ihre geistige Entwicklung vorantreiben, indem sie sich "europäisieren". Zwar behauptet Husserl, dass es "keine Zoologie der Völker" (Hua VI, 320) gibt, aber seine Konzeption kann schwer als vorurteilslos angesehen werden. Zudem stellt sich die Frage, ob diese höchste Stufe an Verantwortung - Verantwortung für das Sein des Menschentums (!), die er wiederum den Philosophen aufbürdet - nicht mit einer intellektuellen Hochmut verbunden ist, der die Verantwortung auf der Ebene des Universellen, Absoluten und der formalen Gesellschaftslehre (Hua XXVII, 50) ansiedelt und damit von den konkreten, sozialen und politischen Verantwortlichkeiten für praktisch-normative Anwendungen der Theorie entbindet. Husserl spricht zwar von Pflichten und der Verantwortung gegenüber Vaterland, Nation und europäischer Gemeinschaft, die wir im alltäglichen Leben haben, aber er unterscheidet diese von der philosophischen Selbstverantwortung (Hua XXIX, 229), die letztendlich als die wahre Verantwortung erklärt wird. Somit wird die faktische Verantwortung in der Transzendentalität der "absoluten Selbstverantwortung" eines "absoluten 'Ich-Selbst'" aufgelöst (Hua XXIX, 165). Auch dort wo sich der späte Husserl konkrete ethische Fragen stellt, kommt er zu Aussagen, die letztendlich die partikulare Verantwortung des Einzelnen im Allgemeinen aufheben:

Alle sind für alles verantwortlich. Und sofern menschliches Leben in einem guten Sinn ein schuldhaftes und sich notwendig als das wissendes ist, können wir auch sagen: Alle 
sind an allem schuld. Es gibt keine bloße Privatethik, sondern Individualethik und Sozialethik, universale Menschheitsethik, sind eine Ethik. Nur das gilt, dass die Fragestellungen von dem jeweilig ethisch sich besinnenden Ich auszugehen haben und für einen jeden das Leben, auch das sozial fungierende Leben, das er verantwortet, das eigene ist und für einen jeden der 'Augenblick', in dem er, aktuell lebend, sich zu entscheiden hat, der seine ist. Er ist der seine, und doch umgreift er - intentional - alle Anderen. Und es gibt vermöge dieser Innerlichkeit, in der alles Leben einig ist, keine Abkapselung irgendeines ethischen Ich und seiner Verantwortungen ${ }^{7}$ (Hua XLII, 476)

\section{Phänomenologische Wege der Verantwortung nach Husserl}

Husserls Tod 1938 hat ihm diverse Probleme und Konfrontationen erspart. Für die Intellektuellen, die den Krieg überlebten, wurde die Frage nach der konkreten Verantwortung zu einer der heftigst debattierten Themen. Ich denke hier nicht nur an Heidegger und an die deutschen Debatten über die Schuldfrage, sondern auch an die Streitgespräche über die Säuberungen in Frankreich. Es gab wenig Intellektuelle wie Julien Benda, die gegen den Verfall der Philosophie in politische Leidenschaften und Verherrlichung des "engagierten" Denkens protestierten und sich für das spekulative Denken, für die universellen, ewigen Werte der Wahrheit und der Gerechtigkeit und ein vereintes übernationales Europa der Vernunft einsetzten (Benda 2006, 5, 70, 94) - ein Standpunkt, der uns irgendwo an Husserl erinnert. Die meisten französischen Philosophen und Schriftsteller verteidigten hingegen die Position, dass die Intellektuellen für alles was sie schreiben verantwortlich sind, sogar mehr als andere. Deshalb insistierten sie in Bezug auf die Nazi-Kollaboration darauf, dass es für die Schriftsteller keine Ausnahmeregelungen geben soll (siehe Suntrup 2011, 102). Mit anderen Worten, nach dem "großen Krieg" wurde klar, dass der Diskurs über die Verantwortung nicht mehr auf das abstrakte Niveau der transzendental-philosophischen Auslegungen bleiben kann, sondern, dass er einer "réflexion seconde" bedarf, d.h. einer Wiedergewinnung des Konkreten und Wiederherstellung des "integralen Ich" in seinen realen Beziehungen (Ricœur 1947, 81). Auf diesem Weg zwischen dem Universalen und dem Partikularen, den Derrida Aporie der Verantwortung nennt (Derrida 1999, 88), bewegen sich meines Erachtens die meisten phänomenologischen Verantwortungsauffassungen nach Husserl, auf die ich hier eingehen möchte.

Die post-husserlschen Konzeptionen der Verantwortung unterscheiden sich, ganz allgemein genommen, in folgenden Punkten von Husserl: Erstens, die Verantwortung ist zwar weiterhin mit einer gewissen Subjektivitätsauffassung verbunden, sie wird jedoch nicht aus der Transzendentalität heraus verstanden, sondern vom Sein oder vom Anderen bzw. vom Fremden her; zweitens, sie wird nicht mit der besonderen Fähigkeit (des europäischen Men-

\footnotetext{
${ }^{7}$ Kursiv von mir, Y.R.
} 
schen) zu rationalem, philosophischem Denken in Bezug gebracht; drittens, sie wird nicht mit einer vornehmlich philosophischen Mission, die aus einer Teleologie der Geschichte abgeleitet wird, verknüpft. Außer in diesen Punkten sind sich die meisten Phänomenologen kaum in etwas einig. Doch wie unterschiedlich sie auch die Verantwortung thematisieren, es zeichnen sich, meines Erachtens, zwei große Interpretationsrichtungen ab.

\subsection{Verantwortung als autonome Entscheidung}

Die Vertreter der ersten Interpretationsrichtung verstehen die Verantwortung als eine aktive, autonome, selbstbestimmte Entscheidung des Menschen die Zuständigkeit für etwas zu übernehmen und bereit sein sich dafür zu verantworten. So zum Beispiel bei Heidegger: Verantwortung heißt für etwas einzustehen, anstatt sich davon zu schleichen und hinter dem Man zu verstecken:

Das Man ist überall dabei, doch so, daß es sich auch schon immer davongeschlichen hat, wo das Dasein auf Entscheidung drängt. Weil das Man jedoch alles Urteilen und Entscheiden vorgibt, nimmt es dem jeweiligen Dasein die Verantwortlichkeit ab. Das Man kann es sich gleichsam leisten, daß 'man' sich ständig auf es beruft. Es kann am leichtesten alles verantworten, weil keiner es ist, der für etwas einzustehen braucht. (Heidegger GA 2, 170)

Die Verantwortung wird auf diese Weise mit der Erschließung der Uneigentlichkeit und der Entschließung zur Übernahme des eigentlichen Seins in Verbindung gebracht. Vom Entschluss dem Man Widerstand zu leisten hängt die Befreiung des Daseins von seiner ursprünglichen Verschuldung im Geworfensein ab. Dieser Entschluss wird nicht einfach rational gefasst, sondern durch das Gewissen vermittelt, welches das Dasein vor sein eigenstes Seinkönnen ruft und es auffordert darauf zu antworten:

Rufverstehend läßt das Dasein das eigenste Selbst aus seinem gewählten Seinkönnen in sich handeln. Nur so kann es verantwortlich sein. (ebd., 382)

Auch Jean-Paul Sartre geht in diese Richtung. Er folgt Heidegger, indem er in Das Sein und das Nichts die Flucht vor der Verantwortung in der Uneigentlichkeit bzw. in der Unaufrichtigkeit/Selbstbetrug (la mauvaise foi) als eine grundlegende Struktur des Für-sichSeins thematisiert (Sartre 1994, 119-160). Der Unterschied liegt darin, dass Sartre die Verantwortung zwar mit dem Gewissen verbindet, jedoch nicht primär von diesem her versteht, sondern von der absoluten Freiheit des Für-sich, die er mit der unausweichlichen Wahl gleichsetzt. Das Für-sich als Bewusstsein ist von vornherein Transzendenz, Freiheit und Wahl und daher auch allzeit verantwortlich und zwar nicht nur für sich selbst, sondern auch für die Anderen: 
Sein ist für Heidegger seine eigenen Möglichkeiten sein, sich sein machen. Und das ist so wahr, daß ich für mein Sein für Andere verantwortlich bin, insofern ich es frei in der Eigentlichkeit (authenticité) oder der Uneigentlichkeit (inauthenticité) realisiere. In voller Freiheit und durch eine ursprüngliche Wahl realisiere ich zum Beispiel mein Mitsein in der Form des 'Man' (...) ich bin meine eigene Eigentlichkeit nur, wenn ich mich unter dem Einfluß des 'Rufs des Gewissens' mit Entschlossenheit auf den Tod hin entwerfe als auf meine eigenste Möglichkeit. In diesem Moment enthülle ich mich mir selbst in der Eigentlichkeit und hebe auch die Anderen mit mir zum Eigentlichen empor. (Sartre 1994, 446)

Die Verantwortung als Konsequenz der freien Wahl erweist sich als absolut und zugleich als absurd. Sie ist absolut, da ich, was immer ich auch tue, eine Wahl treffe; nicht wählen bedeutet zu wählen, dass man nicht wählt. Die Verantwortung ist zugleich absurd, denn ich habe nicht gewählt zu sein, muss jedoch mich und mein Sein ständig wählen und dafür Verantwortung tragen. ${ }^{8}$

Im Gegensatz zu Heidegger bleibt Sartre nicht auf der ontologischen Ebene stehen, sondern entwirft die berühmt gewordene Konzeption des sozialen Engagements als eines Gegenstücks zur "Versuchung der Unverantwortlichkeit" der Schriftsteller und der Intellektuellen (Sartre 1986a, 156 und 1986b). Dabei taucht auch das Thema der "unendlichen" Verantwortung auf (Sartre 1986b, 17 und 1994, 121-122), das später bei Jonas und Levinas einen wichtigen Platz einnimmt und von Ricœur kritisiert wird (Raynova 2016, 90).

Die soziale bzw. geschichtliche Verantwortung als eines Modus menschlicher Selbstbestimmung wird zum speziellen Thema auch für Hannah Arendt, Jan Patočka und Hans Jonas.

Hannah Arendt, die sich mit der persönlichen Verantwortung unter totalitären Verhältnissen beschäftigt, stellt die Frage: Was macht den Unterschied aus zwischen den Dienern des Naziregimes und denen, die sich geweigert haben mitzumachen? (Arendt 2003, 43-44) Die Mitläufer haben in den Kriegsprozessen ihre Schuld geleugnet und behauptet sie selbst hätten verantwortungsvoll gehandelt, da sie ihre Pflicht und die ihnen erteilten Befehle gewissenhaft und gehorsam ausführten, während hingegen die Anderen, die nicht Mitläufer, es sich insofern leicht machten, als sie keine politische Verantwortung übernahmen. Arendts lange und subtile Analysen münden in der These, dass wahre Verantwortung nur durch die Urteilskraft als kritisches, selbständiges Denken möglich ist. Denn Letzteres leitet nicht einfach die eigenen Handlungsentscheidungen von geltenden Normen ab, welche die Machthabenden vorgeben, sondern diese kritisch im inneren Dialog mit sich selbst überprüfen. Gerade in totalitären

\footnotetext{
${ }^{8}$ Die Aktualität dieses Themas in Bezug auf die Urstiftung menschlicher Verantwortung zeigt Artur Bodelderl in seinem Beitrag, in dem die pränatale Situation, in welcher der Mensch über gar keine Sphäre "eigener" Entscheidungen verfügt und demnach völlig unfrei ist, thematisiert wird (Boelderl 2016, 96ff).
} 
Gesellschaften wird dann die politische Verantwortungslosigkeit als ziviler Ungehorsam oder passiver Widerstand zu einer authentischen Form der moralischen Verantwortung (ebd. 44-45).

Jan Patočka, der selbst passiven Widerstand unter dem kommunistischen Regime in der Tschechoslowakei leistete, entfaltet seinerseits die Frage der Verantwortung auf zwei Ebenen, die durch die Oppositionen "Alltägliches - Feierliches" und "Profan - Sakral" charakterisiert sind. Auf der ersten Ebene, diejenige des äußeren Lebens, geht es um die Flucht vor der Verantwortung in das Man (Heidegger), d.h. in die Sachlichkeit des Alltags, oder um die Übernahme der Verantwortung als eine Lebenslast (Patočka 2010, 119). Auf der zweiten Ebene geht es nicht mehr um das Leben selbst, sondern um das unwahre Ich im Bereich der Arbeit und das wahre Ich als Ort der Sorge für die Seele und ihr Heil. Die Verantwortung zeigt sich hier in Bezug auf ein höchstes Seienden, das uns weniger von außen, als von innen beherrscht und als ein "Sich-Öffnen für die Abgründigkeit des Göttlichen und des Menschlichen" (ebd., 128-129). Verantwortung wird nur dann möglich, wenn wir das Dämonische als das Unverantwortliche in den Griff bekommen, es beherrschen, und zugleich uns dem höchsten Seienden in freier Entscheidung unterwerfen. Die Verbindung zwischen dem Sakralen und der Verantwortung, in der Patočka die Quelle des Religiösen sieht, wird von ihm als ein komplexer Werdegang der Geschichte Europas als eine Geschichte der Verantwortung verstanden. Das Zentrum dieses Werdegangs bildet der Übergang von der Äußerlichkeit in der griechischen Philosophie zur Innerlichkeit im Christentum. Die Verinnerlichung der Verantwortung, die dabei stattfindet, kommt durch ein "Zu-sich-selbst-Gelangen in der Wahl" zustande, bei dem die Seele dem Verfall im Dienen des Alltäglichen entgeht, indem sie Widerstand leistet. Die (europäische) Geschichte mit ihren Kriegen verlaufe in diesem ständigen Kampf zwischen Verantwortung und Alltäglichkeit. Gerade im Zeitalter der technischen Zivilisation, in welcher der Mensch entfremdet und "innerlich verelendet" um sein echtes Selbst gebracht ist, bedarf er mehr denn je der inneren Umkehr. Diese zwingt ihn sowohl Verantwortung für die Sinnlosigkeit (ebd., 96), als auch für die "Konflikte von planetarischen Ausmaßen", die aus der zunehmenden Machtentfaltung resultieren, zu übernehmen (ebd., 139).

In ähnlicher Richtung geht auch die Warnung von Hans Jonas vor den Gefahren einer "allmächtig gewordenen technischen Zivilisation" (Jonas 1979, 245), die nicht nur zum Selbstverlust, sondern auch zu einer möglichen Auslöschung der ganzen Menschheit führen könnte. Obwohl Geschichte und Gedächtnis bei Jonas auch eine Rolle spielen (ebd., 200-204, 227-230), ist seine Verantwortungskonzeption nicht vergangenheitsorientiert, sondern vorwiegend auf die zukünftigen Folgen des Handelns ausgerichtet:

Die Zukunft der Menschheit ist die erste Pflicht menschlichen Kollektivverhaltens im Zeitalter modo negativo 'allmächtig' gewordenen technischen Zivilisation. Hierhin ist die Zukunft der Natur als sine-qua-non offenkundig mitenthalten... (ebd., 245) 
Diese Pflicht entspringt aus der "apokalyptischen Situation", welche die Menschen des 20. Jahrhunderts selbst geschaffen haben und die durch eine Überdimensionierung der Macht über die Natur charakterisiert ist. Die technologische Macht, die selbstmächtig geworden ist (Macht zweiten Grades) und dadurch eine Ohnmacht der Kontrolle des Nutzers darstellt, kann nur dialektisch, durch eine andere Macht überwunden werden. Anders gesagt, wir bedürfen einer Macht über die verselbständigte Macht (Macht dritten Grades), um den "vermeinten Besitzer" der Macht aus seiner Knechtschaft zu befreien (ebd., 254). Diese Macht dritten Grades soll durch seine Ethik der Verantwortung, die durch den intrinsischen Wert des Lebens begründet wird, möglich gemacht werden. Sie basiert auf einer Neuformulierung des ethischen Imperativs: "Handle so, dass die Wirkungen deiner Handlung verträglich sind mit der Permanenz echten menschlichen Lebens auf Erden" (ebd., 36). Verantwortung bedeutet in diesem Sinn "die als Pflicht anerkannte Sorge um ein anderes Sein, die bei Bedrohung seiner Verletzlichkeit zur 'Besorgnis' wird" (ebd., 391). Sie ist die Antwort auf die Frage was einem bedrohten verletzlichen Wesen zustoßen würde, wenn ich mich seiner nicht annehme. "Je dunkler die Antwort", betont Jonas, "desto heller gezeichnet die Verantwortung" (ebd.). Die Einbildungskraft, die Heuristik der Furcht, die Ehrfurcht, sowie das Schaudern sind Wege, uns vor den Irrwegen unserer Macht zu schützen. Ähnlich wie Patočka und der frühe Ricœur betont Jonas die Notwendigkeit einer Wiederherstellung der Kategorie des Heiligen:

Die Ehrfurcht allein, indem sie uns ein 'Heiliges', das heißt unter keinen Umständen zu Verletzendes enthüllt (und das ist auch ohne positive Religion dem Auge erscheinbar) wird uns auch davor schützen, um der Zukunft willen die Gegenwart zu schänden, jene um den Preis dieser kaufen zu wollen. (ebd., 393)

In seinen späteren Werken geht Jonas sogar so weit, der schwachen und verletzbaren Kreaturen Gott selbst hinzuzufügen, da dieser sich durch die Schöpfung begrenzt und damit freiwillig entmachtet habe ${ }^{9}$. Etty Hilesum folgend vertritt er die "ketzerische Ansicht", dass "nicht Gott uns helfen kann, sondern wir ihm helfen müssen" (Jonas 1988, 60). Somit wird die ganze Verantwortung für das Gelingen oder Misslingen des "göttlichen Abenteuers" auf den Menschen übertragen:

In unseren unsicheren Händen halten wir buchstäblich die Zukunft des göttlichen Abenteuers auf Erden und wir dürfen Ihn nicht im Stiche lassen, selbst wenn wir uns im Stiche lassen wollten. (Jonas 1987a, 62)

\footnotetext{
${ }^{9}$ In seinem Werk Der Gottesbegriff nach Auschwitz (1987) verneint Jonas Gottes Allmacht und erklärt wieso er nicht in unserer Geschichte eingreift: "Verzichtend auf seine eigene Unverletzlichkeit erlaubte der ewige Grund der Welt, zu sein. Dieser Selbstverneinung schuldet alle Kreatur ihr Dasein und hat mit ihm empfangen, was es von jenseits zu empfangen gab. Nachdem er sich ganz in die werdende Welt hineinbegab, hat Gott nichts mehr zu geben: jetzt ist es am Menschen, ihm zu geben" (Jonas 1987b, 47).
} 
Das Verantwortungskonzept von Jonas ist also viel breiter gefasst als dasjenige des späten Husserl, dem es um die Rettung der Menschheit durch die Vernunft geht, oder bei Heidegger und anderen Phänomenologen, die den Akzent nur auf die Selbstverantwortung legen. Sie stellt die höchst möglichen Ansprüche an den Menschen, wobei die Frage ist, ob das nicht zu einer maßlosen Überforderung führt (Ricœur 1998, 24f; Sommer 1995, 353) ${ }^{10}$, da der Mensch nun an Stelle Gottes, sowohl für alle Übel der Welt einstehen, als auch die Rolle des Schöpfers und Erlösers übernehmen muss (Marquard 1983, 165). In diesem Punkt hat Susanne Moser wohl Recht, wenn sie anmerkt, Jonas stehe "sehr nahe bei Jean-Paul Sartre" (Moser 2016, 64). Der wesentliche Unterschied zwischen Jonas und Sartre wäre, dass wir nach Sartre für alles in der Welt verantwortlich sind, weil es eben keinen Gott gibt, während wir nach Jonas verantwortlich sind, weil Gott sich selbst entmachtet hat, um uns Autonomie und Macht zu verleihen.

\subsection{Die Passion der Verantwortung}

Die Vertreter der zweiten Interpretationsrichtung verstehen die Verantwortung primär als passives Unterworfensein, da wir dem Anspruch des Anderen/Fremden immer schon unterstellt sind. Hier wird nicht nur der Husserl'sche Idealismus, sondern auch der fundamentalontologische Zugang Heideggers überschritten, hinterfragt oder heftig kritisiert ${ }^{11}$. Der Ruf zur Verantwortung kommt nicht mehr vom "Inneren", vom Gewissen her, sondern vom Anderen oder vom Fremden. Paradigmatisch dafür sind die Verantwortungskonzeptionen von Emmanuel Levinas und Jacques Derrida ${ }^{12}$.

Die Levinas'sche Philosophie, die man, Derrida folgend (Derrida 1967, 140), als "Heterologie" bezeichnen kann, steht im Gegensatz sowohl zur Husserl'schen Egologie als auch zu Heideggers Differenzdenken. Denn die Fundamentalfrage für Levinas bezieht sich weder auf das (transzendentale) Subjekt, noch auf den Sinn von Sein, sondern auf den Anderen, dessen Antlitz uns befiehlt nicht zu töten und uns zur Verantwortung aufruft (Levinas 1982a, 91). In einem Gespräch mit Michael Jacob erklärt Levinas, dass, während die abendländische Philosophie sich mit dem "konstruktiven, positiven Subjekt" beschäftigt, bei ihm es darum geht, "daß das Humane mit dieser Verantwortlichkeit für den Anderen und der Sorge für den Anderen anfängt; das Bewußtsein, daß dieses Ich, das da ist, auserwählt ist, verantwortlich zu sein für die Welt, d. h. für all die Anderen in der Welt. Deswegen zitiere ich immer wieder

\footnotetext{
${ }^{10}$ Eine moderate Auffassung der Verantwortung in Anknüpfung an Jonas schlägt Antje Kapust vor (Kapust 2016, 104ff).

${ }^{11}$ Z.B. die Levinas'sche Kritik an Heideggers "ontologischer Imperialismus" oder "Imperialismus des Gleichen" (Levinas 1990, 35, 86).

${ }^{12} \mathrm{Zu}$ diesen Konzeptionen könnte man auch Bernhard Waldenfels' responsive Ethik hinzufügen, die gewisse Ähnlichkeiten mit Levinas' und Derridas' Auffassungen der Verantwortung aufweist (Waldenfels 1994, 2006). Eine Alternative zu diesen Konzeptionen bietet Hans Peter-Krüger (Krüger 2016).
} 
den Satz Dostojewskijs: 'Ein jeder von uns ist verantwortlich für alles und alle und ich selbst mehr als jeder andere"' (Jacob 1994, 43; vgl. Levinas 1974, 186). Levinas zitiert also gerne einen Satz, der auch ein Lieblingssatz von Sartre (Sartre 1986b, 17) und Ricœur (Ricœur 1998, 30) ist, jedoch geht es bei ihm nicht um eine Konstitution des Anderen als ein "anderes Ich" (alter ego), das eben, weil ein Ich, dieselben Rechte und Ansprüche hat wie Ich selbst, denn dies würde wieder zu einer Philosophie des Selben führen. Er betont deshalb immer wieder, dass es ihm um das Erscheinen des Anderen in seiner grundsätzlichen Andersheit und seinem Primat über das Selbst geht, d.h. um die radikale Differenz zwischen mir und dem Anderen/Nächsten (autrui). Denn der Andere wird nicht Anderer durch meinen Bezug zu ihm bzw. durch irgendeine Eigenschaft, die ihn von mir unterscheidet: er bleibt mir gegenüber "unendlich transzendent, unendlich fremd" (Levinas 1990, 211). Im Gegenteil, es ist das Ich das durch den Anderen zum Selbst wird, in dem es ihm antwortet und für ihn Verantwortung übernimmt (Levinas 1990, 194). Der Vorrang des Anderen bedeutet, dass ich ihm schon von vornherein ausgesetzt bin (Levinas 1974, 113), dass es eine vor-ursprüngliche Verantwortung gibt, in die ich geworfen bin, in der ich, biblisch gesprochen, "der Hüter meines Bruders bin" (Levinas 1974, 150 und 211). Es ist eine Verantwortung, die ich nicht gewählt habe, die schon da ist vor meiner Wahl, vor meiner Entscheidung, vor meiner Freiheit. Sie ist da, weil der Andere/Nächste schon da ist und ich ihn erblicke und ihm begegne als ein Gesicht, das mir seine Nacktheit und Verletzlichkeit entblößt und an mich appelliert. Den Anderen/Nächsten erkennen, betont Levinas, heißt einen Hunger erkennen, heißt geben. Doch im Gegensatz zu Jonas und Ricœur, bei denen der Ruf ausschließlich aus dem Verletzlichen und Schwachen ergeht, spricht Levinas auch eine andere Dimension an - die des Höchsten als Herr, Herrscher, Befehlender. Das Erblicken, Transzendieren und Erkennen führt nicht zum Selben auf Augenhöhe, sondern hinauf, zur Transzendenz. Durch das Gesicht des Nächsten, des Bruders, für den ich verantwortlich bin, erkenne ich den Vater und sein Gebot an. In diesem exegetischen Sinne wäre, meines Erachtens, Levinas' zu verstehen, dass "dem Anderen geben", heißt dem Herren (maître, seigneur) geben, dem, den man als "Sie" (vous) in der Dimension der Höhe ("dimension d'hauteur") begegnet (Levinas 1990, 73). Deshalb erläutert er im erwähnten Interview:

Ich behaupte übrigens oft, daß meine ganze Philosophie in dieser Geste zusammengefaßt werden kann: 'Nach Ihnen, mein Herr!' Das Antlitz möchte ich in diesem Sinne verstanden wissen und nicht aufgrund irgendwelcher visueller Kategorien., dass seine ganze Philosophie in dem einen Satz resümiert werden kann: 'Nach Ihnen, mein Herr'. (Jackob 1994, 53; vgl. Levinas 1974, 150).

Dazu kommt, dass die etymologische Verbindung zwischen Anderer (aher) und Verantwortung (acharaiout) im Hebräischen aus der Wurzel ah (Bruder) her zu verstehen ist 
(Chalier 1986, 64). Mit dem Gesicht des Anderen wird mir also eine Verantwortung auferlegt, die von Ihm $(i l)$, dem Höchsten, der Illeität ${ }^{13}$ her abgeleitet wird, der der mir gebietet für den Anderen/Nächsten als Bruder "da" zu sein, koste es, was es wolle. Diese Verletzlichkeit verpflichtet mich sogar dann, wenn ich im Gesicht des Anderen dem Hass eines Verfolgers begegne. Ich muss in diesem Fall von dem Trauma der zu-erleidenden-Schmähung zur Verantwortung übergehen, d.h. zur Sühne (Levinas 1974, 141). Diese umstrittene Levinas'sche Position wird von Judith Butler folgendermaßen interpretiert:

\begin{abstract}
It is surely hard to feel at once vulnerable to destruction by the other and also responsible for the other, and readers of Levinas object all the time to his formulation that we are, all of us, in some sense responsible for that which persecutes us. He does not mean that we bring about our persecution - not at all. Rather, "persecution" is the strange and disconcerting name that Levinas gives to an ethical demand that imposes itself upon us against our will. We are, despite ourselves, open to this imposition, and though it overrides our will, it shows us that the claims that others make upon us are part of our very sensibility, our receptivity, and our answerability. (Butler 2015, 109)
\end{abstract}

Auch wenn Butler hier die metaphysische (religiöse) Dimension der Verantwortung außer Acht lässt und nur die ethische Seite hervorhebt, trifft ihre Interpretation meiner Meinung nach zu, da Levinas ausdrücklich betont, dass die Beziehung zum Anderen immer mit Verantwortung verbunden ist, unabhängig davon, ob wir sie übernehmen oder abweisen, ob wir für ihn etwas tun oder nicht (Levinas 1982a, 93). Anders ausgedrückt, das Ich befindet sich (vor)ursprünglich in einer asymmetrischen Situation zum Anderen als Befehlshaber und Ankläger: es ist "Geisel" (otage) einer Verantwortung für den Anderen, die es nicht gewählt hat, die, noch bevor es sich frei entschieden hat, seine Freiheit in Anspruch nimmt und diese, samt seiner Identität, bestimmt (Levinas 1974, 145, 180). Zugleich suggeriert Levinas, dass diese passive Unterworfenheit durch eine bejahende Antwort - "hier bin ich" für Dich - überschritten werden kann. Indem das Ich antwortet und sich verantwortet, indem es seine Empfangsbereitschaft für den Anderen bezeugt, kann es von der Geisel zum Gastgeber werden (Levinas 1990, 334), was jedoch seine endlose Abhängigkeit vom Anderen nicht aufhebt, sondern nur noch multipliziert (Levinas 1982b, 106).

Nicht weniger radikal scheint Derrida zu sein, obwohl er nach einem Mittelweg zwischen Heidegger und Levinas sucht. Seine Verantwortungsauffassung wird am deutlichsten ausgesprochen in einer Diskussion, die 1985 im Rahmen des internationalen Kongresses über die Krise der Phänomenologie in Wien stattfand. Dort erklärt er wie es mit der Dekonstruktion zu einer Verschiebung des Verantwortngswertes, "von dem zuvor nie die Rede

\footnotetext{
${ }^{13}$ Der Levinas'sche Neologismus illéite, der vom il (er) gebildet wurde, bezeichnet die dritte Person als Er, der absolut Andere, der Abwesende, der Unsichtbare hinter dem Anlitz, d.h. Gott.
} 
gewesen sei", gekommen ist (Derrida 1986, 170). Seit Heidegger sei die Verantwortung nicht mehr diejenige eines transzendentalen Egos oder einer transzendentalen Gemeinschaft mit ihrem Telos der unendlichen Aufgabe, sondern Verantwortung ist nunmehr mit dem Sein und der Differenz verbunden, d.h. mit "etwas, das uns stellt, wovor wir als egologische Subjekte zu antworten haben" (ebd.). Es handle sich dabei nicht mehr um eine Antwort im Sinne einer kritischen Entscheidung oder kritischen Wahl, sondern um eine Thematisierung des Unentscheidbaren. In Anlehnung an Heidegger unterscheidet Derrida zwei Typen der Unentscheidbarkeit: eine, die der Ordnung des Kalküls als etwas Berechenbarem zugehört und eine ganz andere, die des Unbestimmbaren und Ungewissen, das uns verantwortlich macht gegenüber dem, was kein Seiendes ist, sondern Grund des Seins des Seienden. Nur wo diese zweite Art von Unentscheidbarkeit herrsche, könne Verantwortung übernommen werden, andernfalls würde es ja genügen, alle Momente des Kalküls zu kennen, um dann daraus die Konsequenzen zu ziehen. Derrida erläutert das folgendermaßen:

Ich glaube, die Verantwortung beginnt genau dann, wenn man keine Gewißheit mehr hat, vor wem (d. h. vor welcher Person, vor welchem Tribunal oder vor welchem Staat bzw. Gesellschaft, seien sie bestimmt oder nur bestimmbar) man verantwortlich ist. Wüßte ich, vor welchem Gericht ich wofür, für welche Sache, für welche Handlung usf. Rechenschaft abzulegen hätte, so wäre ich nicht mehr verantwortlich. Ich würde lediglich ein Programm anwenden, mich entsprechend dem Gesetz und den Regeln verhalten. (ebd., 174-175)

Derrida fügt hinzu, er verstehe sich zwar gut auf Verpflichtungen und Gesetze - er erfülle seine Lehrpflichten, zahle seine Steuern usw., - aber was das Wesentliche der Verantwortung ausmache, darauf wisse er keine Antwort. Er spitzt diese Ungewissheit des Unbestimmbaren zusätzlich zu, indem er den Heidegger'schen Ruf des Anderen als einen Ruf von Nichts und Niemanden interpretiert:

Ich höre den Ruf zur Verantwortung dann, wenn dieser Ruf, der vom Anderen in seiner Allgemeinheit und Unbestimmtheit ausgeht, nicht mehr als subjektives oder objektives Seiendes bestimmbar ist. Ich bin also nicht verantwortlich vor jemandem im Sinne eines bestimmten Subjekts und für etwas im Sinn eines bestimmten Objekts. Der Ruf der Verantwortung kommt mir vom Sein, insofern das Sein nichts ist, insofern es nicht ein bestimmtes Seiendes ist. (...) Ich glaube, es ist in der Tat die Bedingung der Verantwortung, daß der Ruf von nichts und von niemandem komme. Das gilt ebenso, wenn Levinas sagt, daß mir die Verantwortung vom Anderen zukommt, von meinem Verhältnis zum Anderen, aber vom Anderen, sofern er nicht als Subjekt gefaßt, sondern unendlich transzendent ist. (ebd. 175)

Man kann über diese Interpretation sicherlich diskutieren. Denn Levinas erklärt explizit, dass er den Anderen/Nächsten, der an mich appelliert, sowohl in der Dimension der Höhe, der 
Illeität des Herrn, als auch in derjenigen der Tiefe, d.h. des Bruders als "der Arme, der Fremde, die Witwe, der Waise", auffasst (Levinas 1990, 281). Wichtig hier ist jedoch das, worauf Derrida selbst hinaus will, nämlich, die Grundthese, dass die Verantwortung nicht von irgendwelchen allgemeinen Gesetzen und Normen ableitbar ist und dass es sich um einen singulären Ruf handelt, der so unbestimmt ist, dass es unklar bleibt, woher und von wem er kommt. Deshalb kann die Verantwortung, seiner Meinung nach, philosophisch nicht erfasst werden. Für Derrida ist es zwar gewiss, daß ich vor dem Anderen verantwortlich bin und seinem Ruf zu antworten habe, aber dieser Andere lässt sich nicht bestimmen:

Damit es Verantwortung gibt, muß es Antwort geben. Ich habe zu antworten, habe dem Anderen zu antworten. Aber wenn der Andere anders ist, so deshalb, weil er nicht in philosophisch bestimmbaren Formen und Begriffen antizipierbar ist. Der Andere (der oder das Andere) ist das, was mich zur Rede stellen kann in Formen, die absolut nicht vorwegzunehmen sind. Es ist da, und es ist jedesmal kein einzelner Anderer, ein einzigartiges Anderes. Ich bin nicht, wie vor Gerichten, verantwortlich vor allgemeingeltenden Gesetzen, ich bin verantwortlich vor dem absolut singulären, unreduzierbaren Ruf, der mir vom Anderen kommt. Das ist eine Beziehung, die philosophisch nicht bestimmbar ist, aus keinerlei Code der Verantwortung abgeleitet werden kann. (ebd.)

Auf die Frage, worin denn die Verantwortung der Dekonstruktion bestehe, antwortet Derrida, dass er mittels der Dekonstruktion die Verschleierung der Verantwortung durch gewisse Philosophemen aufzeigen möchte, die sie als etwas darstellen, was das Subjekt durch den freien Willen auf sich nehmen könne. Die Verantwortung sei aber eher im Levinas'schen Sinne zu verstehen, nämlich als etwas, das über mich hereinbricht, dem ich immer ungleich und unterstellt bin (ebd., 176). Was dies konkreter bedeutet kann man aus seinen anderen Schriften entnehmen, in denen es darum geht, die Verantwortung als eine Art Aporie zu enthüllen (Derrida 1991, 43). Dort wird gezeigt, dass die Verantwortung sich weder aus allgemeinen Gesetzen, Normen, Programmen, noch aus freien, nicht normierten Entscheidungen ableiten lasse. Wenn man nach vorgeschriebenen Gesetzen handelt, dann ist das für Derrida schlicht Pflichterfüllung oder Gehorsam, aber keine Verantwortung und, umgekehrt, wenn man nur seinem eigenen Willen folgt, dann ist es Willkür. Die Aporetizität der Verantwortung zeigt sich insbesondere in Bezug zum Wissen: man kann eine verantwortliche Entscheidung nicht fällen, ohne zu wissen was man tut, aus welchem Grunde und mit welcher Absicht man handelt; zugleich ist eine Entscheidung, die einem solchen Wissen folgt, keine verantwortliche, sondern nur "die technische Inbetriebnahme eines kognitiven Dispositivs" (Derrida 2010, 202). Wenn aber die Verantwortung eine solche "mission impossible" darstellt, wenn sie so unmöglich zu bestimmen ist, dann taucht die Frage auf, ob Derrida nicht letztendlich etwas Ähnliches tut wie Husserl, wenn er die Verantwortung in ein so entlegenes 
Reich verlegt (ins Nichts), dass sie jeder praktischen Zuständigkeit enthoben wird. Danach sieht es aus, wenn man Hannah Arendt folgend zwischen der Urteilskraft als eines selbständigen, kritischen Denkens und dem Kalkül als Berechnung von Vorgangsweisen unterscheidet; dann wären Wissen und Unwissen, Macht und Ohnmacht keine Aporien, sondern Schlüsselbegriffe für das Erfassen von Verantwortung und Unverantwortlichkeit. Vielleicht kann man aber Derrida auch anders interpretieren. Denn in seiner Patočka-Auslegung schafft er es das Unbestimmte doch so zu umreißen, dass es aus dem "Nichts" des Seins in die Konturen des Geschichtlichen hervortritt:

Die Ausübung der Verantwortung scheint keine andere Wahl zu lassen als die unbequemste, die es gibt, die des Paradoxes, des Ketzertums und des Geheimnisses. Schwerwiegender noch, sie muss stets die Gefahr der Konversion und der Apostasie in Kauf nehmen: keine Verantwortung ohne den dissidenten und erfinderischen Bruch mit der Tradition, der Autorität, der Orthodoxie, der Regel, der Doktrin. (Derrida 2010, 205)

So gefasst, ist nicht dieser dissidente Bruch derselbe Widerstand, derselbe zivile Ungehorsam, von dem Arendt meinte, er sei die ethische Schwelle der wahren Verantwortung?

Yvanka B. Raynova

\section{Literaturangaben}

Arendt, Hannah. Responsibility and Judgement. New York: Schocken Books, 2003.

Beauvoir, Simone. "Für eine Moral der Doppelsinnigkeit", in idem. Soll man de Sade verbrennen? Drei Essays zur Moral des Existentialismus. Rowohlt: Reinbek bei Hamburg 1989, 77-192.

Benda, Julien. La trahison des clercs. (Coll. Les classiques des sciences sociales, http://classiques.uqac.ca/, version numérique par Pierre Palpant). Québec: Université du Québec, 2006.

Boelderl, Artur R. " Das Fleisch der Verantwortung", Labyrinth, Vol. 18, No. 1 (2016): 95-113.

Brentano, Franz. "Über die Entmutigung auf philosophischem Gebiete", in idem. Über die Zukunft der Philosophie. Leipzig: Felix Meiner, 1929, 83-100.

Buckley, Philip. "Self-Responsibility and Responsibility for Others," Labyrinth, Vol. 18, No. 1 (2016): 31-45.

Chalier, Catherine. Les Matriarches, Paris: Cerf, 1986.

Butler, Judith. Notes Toward a Performative Theory of Assembly. Harvard University Press, 2015.

Derrida, Jacques. "Fragen an die Phänomenologie - Abschied vom Prinzipiellen?", in Benedikt, Michael und Rudolf Burger (Hrsg.). Die Krise der Phänomenologie und die Pragmatik des Wissenschaftsfortschritts. Wien: Edition S, Verlag der Österreichischen Staatsdruckerei, 1986, 169-179.

Derrida, Jacques. De l'esprit. Heidegger et la question. Paris: Éditions Galilée, 1987. 
Derrida, Jacques. L'autre cap. Suivie de La démocratie ajournée. Paris: Minuit, 1991.

Derrida, Jacques. Donner la mort. Paris: Galilée, 1999.

Derrida, Jacques. "Ketzertum, Geheimnis und Verantwortung: Patočkas Europa", in Jan Patočka. Ketzerische Essays zur Philosophie der Geschichte. Berlin: Suhrkamp, 2010, 181-211.

Heidegger, Martin. Sein und Zeit (GA Bd. 2), Vittorio Klostermann, 1977.

Husserl, Edmund. Die Krisis der Europäischen Wissenschaften und die transzendentale Phänomenologie. Eine Einleitung in die phänomenologische Philosophie (Hua Bd.VI). 2. Aufl., Dordrecht/ Boston/ Lancaster: Nijhoff, Kluwer Academic Publishers, 1976.

Husserl, Edmund. Erste Philosophie (1923/24), Erster Teil (Hua Bd.VII). Dordrecht/ Boston/ Lancaster: Nijhoff, Kluwer Academic Publishers, 1956.

Husserl, Edmund. Erste Philosophie (1923/24), Zweiter Teil (Hua Bd.VIII). Dordrecht/ Boston/ Lancaster: Nijhoff, Kluwer Academic Publishers, 1959.

Husserl, Edmund. "Philosophie als strenge Wissenschaft", in idem. Aufsätze und Vorträge (1911-1921), (Hua Bd. XXV). Dordrecht/ Boston/ Lancaster: Nijhoff, Kluwer Academic Publishers, 1987, 3-62.

Husserl, Edmund. Aufsätze und Vorträge 1922-1937 (Hua Bd. XXVII). Dordrecht/ Boston/ Lancaster: Kluwer Academic Publishers, 1989.

Husserl, Edmund. Die Krisis der Europäischen Wissenschaften und die transzendentale Phänomenologie. Ergänzungsband. Texte aus dem Nachlass 1934-1937 (Hua Bd. XXIX). Dordrecht/ Boston/ Lancaster: Kluwer Academic Publishers, 1993.

Husserl, Edmund. Grenzprobleme der Phänomenologie. Analysen des Bewusstseins und der Instinkte. Metaphysik. Späte Ethik. Texte aus dem Nachlass 1908-1934 (Hua Bd. XLII). Dordrecht/ Heidelberg, New York/ London: Springer, 2013.

Jacob, Michael. "Gespräch mit Emmanuel Lévinas", in idem. Aussichten des Denkens. München: Fink, 1994, 41-54.

Jonas, Hans. Prinzip Verantwortung. Versuch einer Ethik für die technologische Zivilisation. Frankfurt am Main: Suhrkamp, 1979.

Jonas, Hans. Zwischen Nichts und Ewigkeit. Drei Aufsätze zur Lehre vom Menschen. Göttingen: Vandenhoeck \& Ruprecht, 1987a.

Jonas, Hans. Der Gottesbegriff nach Auschwitz. Eine jüdische Stimme. Frankfurt am Main: Suhrkamp, $1987 b$.

Jonas, Hans. Materie, Geist und Schöpfung. Kosmologischer Befund und kosmogonische Vermutung. Frankfurt am Main: Suhrkamp, 1988.

Kant, Immanuel. Kritik der Urteilskraft. (Immanuel Kant: Gesammelte Schriften, Akademieausgabe, Bd.5). Berlin: De Gruyter, 1963.

Kant, Immanuel. Idee zu einer allgemeinen Geschichte in weltbürgerlicher Absicht (Immanuel Kant: Gesammelte Schriften, Akademieausgabe, Bd.8). Berlin: De Gruyter, 1969.

Kapust, Antje. Verantwortung angesichts humanbiologischer Herausforderungen", Labyrinth, Vol. 18, No. 1 (2016): 114-129.

Krüger, Hans-Peter. " Verantwortung für die Fraglichkeit des Menschen? Zum Status der Philosophischen Anthropologie", Labyrinth, Vol. 18, No. 1 (2016): 46-57.

Levinas, Emmanuel. Totalité et infini. Essais sur l'extériorité. Paris: Le Livre de poche, [1971] 1990.

Levinas, Emmanuel. Autrement qu'être ou au-delà de l'essence. La Haye: Martinus Nijhoff, 1974. 
Levinas, Emmanuel. Ethique et infini. Paris: Le Livre de poche, 1982a.

Levinas, Emmanuel. L'au-delà du verset. Paris: Minuit, 1982b.

Malina, Peter. "Der Österreichische Kulturbund. Ergebnisse einer fragmentarischen Spurensicherung", in Benedikt, Michael und Rudolf Burger (Hrsg.). Die Krise der Phänomenologie und die Pragmatik des Wissenschaftsfortschritts. Wien: Edition S, Verlag der Österreichischen Staatsdruckerei, 1986, 250-272.

Marquard, Odo. "Theodizee, Geschichtsphilosophie, Gnosis", in Norbert W. Bolz und Wolfgang Hübner (Hrsg.). Spiegel und Gleichnis. Festschrift für Jacob Taubes. Würzburg: Königshausen und Neumann, 1983, 160-167.

Mounier, Emmanuel. Euvres, tome 1. Paris: Seuil, 1961.

Mounier, Emmanuel. Euvres, tome 3. Paris: Seuil, 1962.

Moser, Susanne. "Verantwortung im Spannungsfeld zwischen Machtentfaltung und Verletzlichkeit: Die Umkehr des Verantwortungsverständnisses bei Hans Jonas", Labyrinth, Vol. 18, No. 1 (2016): 58-78.

Murray, Michael. "Husserl and Heidegger: Constructing and Deconstructing Greek Philosophy," Review of Metaphysics, XLI (1988): 501-518.

Patočka, Jan. Die Bewegung der menschlichen Existenz. Phänomenologische Schriften II. Stuttgart: Klett-Cotta, 1991.

Patočka, Jan. Ketzerische Essays zur Philosophie der Geschichte. Berlin: Suhrkamp, 2010.

Platon. Sämtliche Werke Bd.2: Lysis, Symposion, Phaidon, Kleitophon, Politeia, Phaidros. Übers. von Friedrich Schleiermacher. Reinbek bei Hamburg: Rowohlt, 2004.

Raynova, Yvanka B. "Das Selbst und die Gegenwart der Verantwortung", Labyrinth, Vol. 18, No. 1 (2016): 79-94.

Ricœur, Paul. Die Fehlbarkeit des Menschen. Freiburg/München: Alber, 1971.

Ricœur, Paul. A l'école de la phénoménologie. Paris: Vrin, 1986, 21-57.

Ricœur, Paul. "Responsabilité : limitée ou illimitée ?" in Coll. (ed.). Le crime contre l'humanité : mesure de la responsabilité ? Paris : CERP, 1998, 24-25.

Sartre, Jean-Paul. Das Sein und das Nichts. Reinbek bei Hamburg: Rowohlt, 1994.

Sartre, Jean-Paul. "Vorstellung von Les Temps modernes", in idem. Schriften zur Literatur 1. Reinbek bei Hamburg: Rowohlt, 1986a, 156-170.

Sartre, Jean-Paul. "Die Verantwortlichkeit des Schriftstellers", in idem. Schriften zur Literatur 4. Reinbek bei Hamburg: Rowohlt, 1986b, 17-38.

Sartre, Jean-Paul. Der Existentialismus ist ein Humanismus, in idem. Philosophische Schriften 1. Reinbek bei Hamburg: Rowohlt, 1994,117-155.

Sommer, Andreas Urs. "Gott als Knecht der Geschichte. Hans Jonas' 'Gottesbegriff nach Auschwitz'". In: Theologische Zeitschrift, 51 (1995): 340-356.

Suntrup, Jan Christoph. Formenwandel der französischen Intellektuellen. Eine Analyse ihrer gesellschaftichen Debatten von der Libération bis zur Gegenwart. Münster: LIT Verlag, 2010.

Waldenfels, Bernard. Antwortregister. Frankfurt am Main: Suhrkamp, 1994.

Waldenfels, Bernard. Schattenrisse der Moral. Frankfurt am Main: Suhrkamp, 2006. 